\title{
Évaluation des apprentissages et numérique : où en est-on?
}

\section{Assessing using digital technologies: Where do we stand?}

\section{Evaluación de los aprendizajes y tecnologías digitales: estado de la cuestión}

https://doi.org/10.52358/mm.vi9.277

RESPONSABLE DU NUMÉRO

Marie-Hélène Hébert, professeure

Université TÉLUQ, Canada

marie-helene.hebert@teluq.ca

RÉSUMÉ

Dans le contexte de la pandémie de COVID-19 qui a entraîné des changements dans la manière d'évaluer les apprentissages des apprenants, ce neuvième numéro de la revue Médiations et médiatisations remet en question la place que peut prendre le numérique dans l'évaluation pour faciliter la tâche des enseignants. Regroupant 14 contributions de formes et de contextes variés, il offre aux lecteurs de quoi inspirer leurs pratiques évaluatives ou travaux de recherche sur la question de l'évaluation.

Mots-clés : évaluation, numérique, enseignement à distance

ABSTRACT

In the context of the COVID-19 pandemic, which has led to changes in the way assessment is managed, this $9^{\text {th }}$ issue of the journal Mediations and mediatizations questions the role of 
digital technologies in assessment in order to support teachers in the heavy task they face. The 14 articles in this issue offer readers inspiration for research and practice in assessment.

Keywords: assessment, digital technologies, distance education

\section{RESUMEN}

En el contexto de la pandemia de COVID-19, que ha provocado cambios en la forma de evaluar el aprendizaje de los estudiantes, este noveno número de la revista Mediaciones y mediatizaciones cuestiona el lugar que puede ocupar la tecnología digital en la evaluación para facilitar la tarea de los docentes. Con 14 contribuciones de diversas formas y contextos, el número ofrece a los lectores inspiración para sus prácticas de evaluación o para la investigación en torno a la evaluación.

Palabras clave: evaluación, tecnologías digitales, educación a distancia

Tâche difficile au dire de plusieurs, l'évaluation des apprentissages exige beaucoup des enseignants, peu importe leur niveau d'enseignement. Parmi les tâches qui leur incombent, il leur faut notamment planifier l'évaluation, concevoir et administrer des activités d'évaluation aux apprenants, corriger les copies, identifier leurs forces et leurs difficultés, communiquer les résultats de l'évaluation et mettre en place des actions de remédiation pour soutenir les apprenants dans leurs apprentissages. La complexité du geste évaluatif conduit à s'interroger sur la place du numérique en évaluation des apprentissages : dans quelle mesure peut-il soutenir les enseignants dans la lourde tâche qui leur incombe?

L'interaction du numérique avec l'évaluation ne date pas d'hier. Pour s'aider à la tâche, les enseignants ont recours depuis de nombreuses années dans leurs classes aux technologies de l'information et de la communication (TIC) pour évaluer les apprentissages des apprenants. Par TIC, il faut comprendre cet " ensemble d'outils et de ressources technologiques permettant de transmettre, enregistrer, créer, partager ou échanger des informations " (Institut de statistique de I'UNESCO, 2010, p. 130). Ce qui a donné lieu et accès à une multitude d'outils comme les questionnaires informatisés pour collecter des données d'apprentissage chez les apprenants.

Plus récemment, la pandémie de COVID-19 a conduit les enseignants à revoir les façons d'évaluer les apprentissages pour les adapter à la distance et a suscité chez plusieurs d'entre eux une panoplie d'interrogations sur l'usage du numérique dans l'évaluation (Papi, Gérin-Lajoie et Hébert, 2020) : est-il possible de transposer à distance les activités d'évaluation habituellement réalisées en présence? Comment créer une activité d'évaluation à distance? Comment profiter de la distance pour proposer d'autres types d'activités d'évaluation? Comment contrer le plagiat et la tricherie dans la réalisation des activités d'évaluation à distance? À ces questions s'ajoutent aussi celles liées à la diffusion des consignes, au soutien à apporter aux apprenants pendant la passation et à la rétroaction à leur offrir. 
C'est dans ce contexte qu'a été lancé l'appel à textes pour le neuvième numéro de la revue Médiations et médiatisations sur la contribution du numérique pour évaluer les apprentissages des apprenants. Signe que l'évaluation par le numérique interpelle, les auteurs ont été nombreux à se manifester! Riche de 14 contributions qui amènent les lecteurs à réfléchir aux formes, aux leviers et aux défis de l'évaluation par le numérique, le numéro s'articule comme suit : 2 articles de recherche, 2 synthèses de travaux d'étudiants, 5 articles de praticiens, 3 articles de discussions-débats, 1 article d'entretien et 1 note de lecture.

Le numéro s'ouvre sur deux articles de recherche qui s'inscrivent à l'intersection même de l'évaluation et du numérique. Tout d'abord, Sophie Nadeau-Tremblay, Mélanie Tremblay, Thérèse Laferrière et Stéphane $\underline{\text { Allaire }}^{1}$ s'interrogent, dans leur article, sur les enjeux et les défis de l'accompagnement d'enseignants dans l'évaluation des apprentissages à l'aide du numérique. Ensuite, l'équipe composée de Julie Lyne Leroux et Jean-Marc Nolla ${ }^{2}$ présente les résultats d'une recherche dont l'objectif visait à identifier les pratiques d'évaluation des apprentissages à distance mises en œuvre par des enseignants du collégial.

Inscrits dans la rubrique "Synthèses de travaux d'étudiants ", les deux articles suivants sont l'œuvre de Jérémie Bisaillon et Edith Potvin-Rosselet, accompagnés de la professeure Diane Leduc. Le premier article $^{3}$, situé dans le contexte d'un cours de troisième cycle universitaire, présente les étapes qui ont conduit au développement d'une e-évaluation dynamique formative, de son design à sa mise à l'essai auprès d'étudiants. Le second article ${ }^{4}$ 'intéresse quant à lui à la façon de documenter et d'analyser les pratiques d'évaluation numérique chez le personnel enseignant. Comme solution, les auteurs proposent la création d'un outil d'auto-observation et de réflexion et exposent son processus d'élaboration.

Les cinq textes qui suivent s'inscrivent dans la rubrique consacrée aux articles de praticiens où la question de l'évaluation et du numérique reçoit, ici encore, toute l'attention qu'elle mérite. La rubrique s'ouvre sur une contribution de Yann Verchier et Christelle Lison ${ }^{5}$ dans laquelle les auteurs font voir différentes stratégies d'évaluation qui ont été implantées dans le contexte de la crise sanitaire, comme les évaluations asynchrones et le dépôt de devoirs en ligne. Vient ensuite un texte de Christine Faller et de Laurent Heiser ${ }^{6}$ qui présente et analyse un outil de formation et d'évaluation, le CurriQvidéo, qui amène les étudiants à produire une vidéo narrant le déroulé d'une séquence ou d'un projet pédagogique. Dans le troisième texte, Valérie Wathelet et Sandrine Vieillevoye ${ }^{7}$ abordent la question de l'évaluation à distance vécue par les étudiants en situation de handicap à travers les résultats d'une enquête menée en 2021 après d'étudiants. Puis, le quatrième texte, rédigé par Myriam Girouard-Gagné et Micheline Joanne Durand ${ }^{8}$, illustre, dans le contexte d'un cours universitaire de deuxième cycle, la mise en place de pratiques pédagogiques et évaluatives inclusives pour tenir compte des besoins de tous les étudiants. Enfin, dans le cinquième texte, Anastassis Kozanitis, Patrice Farand et Yves Boudreault numérique qui permet de suivre, d'évaluer et de présenter les compétences d'étudiants inscrits dans une université d'ingénierie.

\footnotetext{
1 https://doi.org/10.52358/mm.vi9.249

2 https://doi.org/10.52358/mm.vi9.254

${ }^{3}$ https://doi.org/10.52358/mm.vi9.252

${ }^{4}$ https://doi.org/10.52358/mm.vi9.243

${ }^{5}$ https://doi.org/10.52358/mm.vi9.239

${ }^{6}$ https://doi.org/10.52358/mm.vi9.242

${ }^{7}$ https://doi.org/10.52358/mm.vi9.238

${ }^{8}$ https://doi.org/10.52358/mm.vi9.255

${ }^{9}$ https://doi.org/10.52358/mm.vi9.250
} 
Le numéro fait ensuite place à trois articles de discussions-débats qui proposent des réflexions sur l'évaluation et le numérique. Le premier texte, celui de Marie-Hélène Hébert et de Sylvie Fontaine ${ }^{10}$, met en lumière l'évaluation authentique comme manière d'apprécier les apprentissages des apprenants à distance dans le contexte où l'épreuve papier-crayon ne devient plus possible. Le second texte, de Venus $\underline{\text { Darius }}^{11}$, fait voir sous la forme d'un récit d'enquête des défis que pose l'évaluation par le numérique, notamment celui de l'inégalité numérique parmi les étudiants d'un cours de deuxième cycle universitaire. Enfin, pour clore la rubrique, le texte de Nadine Talbot et Christophe Gremion ${ }^{12}$ offre des pistes de réponses à un questionnement qui en intéresse plus d'un : comment évaluer réellement ce qui doit être enseigné?

Puis, comme treizième et avant-dernier texte se trouve un article sous forme d'entretien de Marie-Josée Dubois, Marie-Ëve Desrochers et Isabelle Nizet ${ }^{13}$ dans lequel les auteures reviennent sur la conception et l'expérimentation d'une situation d'évaluation par les pairs en contexte de formation à distance et en détaillent les bénéfices et les limites pédagogiques.

Le numéro se conclut avec une note de lecture de Naomie Fournier Dubé ${ }^{14}$ qui invite à la lecture de l'ouvrage intitulé Évaluation des apprentissages en formation à distance: Enjeux, modalités et opportunités de formation en enseignement supérieur, paru en 2021, sous la direction de France Lafleur, Jean-Marc Nolla et Ghislain Samson.

Sur ce, bonne lecture!

\section{Liste de références}

Institut de statistique de I'UNESCO (2010). Guide de mesure pour l'intégration des technologies de l'information et de la communication (TIC) en éducation. Montréal, Canada : UNESCO - ISU.

Papi, C., Gérin-Lajoie, S. et Hébert, M.-H. (2020). Se rapprocher de l'évaluation à distance : Dix pistes de réponse. Évaluer. Journal international de recherche en éducation et formation, numéro hors-série 1, 201-206.

\footnotetext{
${ }^{10} \mathrm{https://doi.org/10.52358/ \textrm {mm } . v i 9 . 2 6 5}$

${ }^{11}$ https://doi.org/10.52358/mm.vi9.245

${ }^{12}$ https://doi.org/10.52358/mm.vi9.244

${ }^{13}$ https://doi.org/10.52358/mm.vi9.253

${ }^{14}$ https://doi.org/10.52358/mm.vi9.247
} 